\title{
Article \\ Maternal Vaginal Colonization and Extended-Spectrum Beta-Lactamase-Producing Bacteria in Vietnamese Pregnant Women
}

\author{
Nguyen Thanh Viet ${ }^{1,+}$, Vu Van Du ${ }^{2,+}{ }^{+}$, Nghiem Duc Thuan ${ }^{3}$, Hoang Van Tong ${ }^{1} \mathbb{D}$, Nguyen Linh Toan ${ }^{4,5}$, Can Van \\ Mao ${ }^{5}$, Nguyen Van Tuan ${ }^{6}$, Srinivas Reddy Pallerla ${ }^{7}\left(\mathbb{D}\right.$, Dennis Nurjadi ${ }^{8}\left(\mathbb{D}\right.$, Thirumalaisamy P. Velavan ${ }^{7,9, *}{ }^{\mathbb{D}}$
} and Ho Anh Son 1,*

Citation: Viet, N.T.; Van Du, V.; Thuan, N.D.; Van Tong, H.; Toan, N.L.; Van Mao, C.; Van Tuan, N.; Pallerla, S.R.; Nurjadi, D.; Velavan, T.P.; et al. Maternal Vaginal Colonization and Extended-Spectrum Beta-Lactamase-Producing Bacteria in Vietnamese Pregnant Women. Antibiotics 2021, 10, 572. https:// doi.org/10.3390/antibiotics10050572

Academic Editors: Krisztina M. Papp-Wallace and Nicholas Dixon

Received: 24 March 2021

Accepted: 11 May 2021

Published: 13 May 2021

Publisher's Note: MDPI stays neutral with regard to jurisdictional claims in published maps and institutional affiliations.

Copyright: (c) 2021 by the authors. Licensee MDPI, Basel, Switzerland. This article is an open access article distributed under the terms and conditions of the Creative Commons Attribution (CC BY) license (https:/ / creativecommons.org/licenses/by/ $4.0 /)$.
1 Institute of Biomedicine and Pharmacy, Vietnam Military Medical University, Hanoi 121-08, Vietnam; nguyenthanhviet@vmmu.edu.vn (N.T.V.); hoangvantong@vmmu.edu.vn (H.V.T.)

2 National Hospital of Obstetrics and Gynecology, Hanoi 110-02, Vietnam; dutruongson@gmail.com

3 ENT Department, 103 Military Hospital, Vietnamese Military Medical University, Hanoi 121-08, Vietnam; thuanbm6@gmail.com

4 Department Post-Graduate Training Management, Vietnamese Military Medical University, Hanoi 121-08, Vietnam; toannl@vmmu.edu.vn

5 Department of Pathophysiology, Vietnamese Military Medical University, Hanoi 121-08, Vietnam; canvanmao2011@gmail.com

6 Department of Rehabilitation, Vietnamese Military Medical University, Hanoi 121-08, Vietnam; nguyentuan.351975@gmail.com

7 Institute of Tropical Medicine, Universitätsklinikum Tübingen, Wilhelmstrasse 27, 72074 Tübingen, Germany; srinivas-reddy.pallerla@uni-tuebingen.de

8 Department of Infectious Diseases, Medical Microbiology and Hygiene, University Hospital Heidelberg, Im Neuenheimer Feld 324, 69120 Heidelberg, Germany; dennis.nurjadi@uni-heidelberg.de

9 Vietnamese-German Centre for Medical Research (VG-CARE), Hanoi 116-10, Vietnam

* Correspondence: velavan@medizin.uni-tuebingen.de (T.P.V.); hoanhsonhp@gmail.com (H.A.S.); Tel.: +49-7071-2985981 (T.P.V.); +84-978-437-229 (H.A.S.)

+ These authors contributed equally to this work.

Abstract: Extended-spectrum $\beta$-lactamase-producing Enterobacterales (ESBL-E) resistance to commonly prescribed drugs is increasing in Vietnam. During pregnancy, ESBL-E may predispose women to reproductive tract infections and increases the risk for neonatal morbidity. Vaginal colonization and infections by Escherichia coli and Klebsiella pneumoniae are seldom studied in Vietnam. In this study, we investigated ESBL-producing Enterobacterales in the birth canal of pregnant women. Between 2016 and 2020, vaginal swabs were collected from 3104 pregnant women (mean gestational age of 31 weeks) and inoculated onto MacConkey agar plates. Colonies were subjected to direct identification and antimicrobial susceptibility testing using the VITEK ${ }^{\circledR}-2$ automated compact system and disk diffusion. ESBL production was determined phenotypically. E. coli, Klebsiella species were identified in $30 \%(918 / 3104)$ of the vaginal swabs, with E. coli being the most common (73\%; 667/918). ESBLproduction was detected in $47 \%(432 / 918)$ of Enterobacterales, with frequent multidrug-resistant phenotype. The overall prevalence of carbapenem resistance was low (8\%). Over $20 \%$ of Klebsiella spp. were carbapenem-resistant. Pregnant women had a high prevalence of colonization and may transmit ESBL-E to neonates at birth, an important risk factor to be considered. The high rate of ESBL-producers and carbapenem resistance in Enterobacterales in Vietnam emphasizes the need for consequent surveillance and access to molecular typing.

Keywords: pregnant women; Enterobacterales; antimicrobial resistance; Vietnam; extended-spectrum beta-lactamase; Escherichia coli; Klebsiella pneumoniae; carbapenem resistance 


\section{Introduction}

Antimicrobial resistance (AMR) is increasingly recognized as a global public health issue in many countries. With a population of more than 96 million and a high burden of infectious diseases, Vietnam faces a significant increase in AMR in the last years.

In low and middle-income countries, antibiotic resistance is increasing with high morbidity and mortality, but resistance data has been poorly documented. The WHO recently declared that the group of bacteria most commonly causing hospital-acquired infections (HAIs) in Asia as priority 1 includes Escherichia coli and Klebsiella pneumoniae [1]. Extended-spectrum $\beta$-lactamases producing Enterobacterales (ESBL-E) infections are increasing in Asia, with E. coli and K. pneumoniae being the predominant contributors of ESBL-E in Vietnam [2].

Reports from 2002 until 2011 suggest an exponential increase in resistance due to ESBLs ESBL from 39 to 55\% in Southeast Asia [3]. In particular, the Philippines and Vietnam have reported a high burden of ESBL-producing E. coli infections of $59 \%$ and $81 \%$, respectively [4]. A point prevalence survey from Vietnamese pediatric hospitals shows $30 \%$ of patients from Vietnamese intensive care units across 16 hospitals had HAIs [5]. In addition, evident that Vietnamese neonatal patients with culture-confirmed Gram-negatives from HAIs had a $50 \%$ case fatality rate [6].

Maternal colonization with group B streptococci (GBS) and Enterobacterales is considered a significant risk factor for the acquisition of neonatal sepsis [7]. While AMR is not a major problem for GBS [8], resistance to beta-lactams in Enterobacterales is emerging and poses a clinical challenge. Among all antibiotic substances, beta-lactams are considered a safe option to treat infections during pregnancy, backed with good-quality evidence [9]. Colonized mothers may transmit ESBL-E to neonates at birth. Thus, colonization by ESBL-producers is an important risk factor in pregnant women [10]. Colonization rates of ESBL-E in pregnant women vary from 3-15\% [10-12]. In particular, maternal vaginal colonization has repeatedly been demonstrated as a risk factor for transmission and hence can cause neonatal sepsis $[7,9,13]$. The emergence of ESBL-producers, especially in E. coli and K. pneumoniae as vaginal colonizers is alarming and of clinical relevance due to limited antibiotic options to treat pregnant women and neonatal early onset sepsis [14].

In Vietnam, the incidence of ESBL-producing strains among clinical E. coli and Klebsiella spp. isolates in vaginal swabs of pregnant women have not been studied. The knowledge of the burden of colonization in pregnant women and the extent of maternal-neonatal transmission is important for AMR surveillance and subsequent prevention strategies [15]. In this context, this study aimed to determine the incidence of ESBL-E in pregnant women attending the national hospital in the last five years (2016-2020).

\section{Results}

\subsection{Baseline Characteristics of Enrolled Participants}

A total of 3863 pregnant women with a mean age of 30 years (17-46) and a mean gestational age of 31 weeks (range 16-41 weeks) were enrolled in the study. Vaginal swab specimens were collected from 3104 pregnant women and were subsequently investigated (Table 1).

\subsection{ESBL-E Carriage in Pregnant Women}

A total of 3104 vaginal swabs from pregnant women were analyzed, and 918 (30\%) isolates were positive for E. coli and Klebsiella spp. Among them, E. coli was predominant $(n=667 / 3104 ; 21 \%)$, followed by K. pneumoniae $(n=243 / 3104 ; 8 \%)$, and K. oxytoca $(n=8 / 3104 ; 0.3 \%)$. The rates of colonization by ESBL-positive and ESBL-negative Enterobacterales in all swab samples were $14 \%(n=432)$ and $16 \%(n=486)$, respectively. ESBL production was detected in $47 \%(432 / 918)$. Among the 432 ESBL-positive isolates, E. coli was predominant $(n=340 / 432 ; 79 \%)$, followed by K. pneumoniae $(n=88 / 432 ; 20 \%$ ), and $K$. oxytoca $(n=4 / 432 ; 1 \%)$. There were no significant differences in the distribution of $E$. coli and Klebsiella spp. between the ESBL-positive and negative isolates (Table 2). 
Table 1. Baseline characteristics of enrolled participants.

\begin{tabular}{|c|c|}
\hline Characteristics & $n(\%)$ \\
\hline Enrolled & 3863 \\
\hline Sample collected & 3104 \\
\hline Positive for Enterobacterales & $918(30)$ \\
\hline Mean age (range) & $30(17-46)$ \\
\hline Gestational age in weeks (range) & $31(16-41)$ \\
\hline \multicolumn{2}{|c|}{918 individuals (positive isolates) } \\
\hline High risk of preterm birth & $150(16)$ \\
\hline Stillbirth & $23(3)$ \\
\hline Primiparous & $284(31)$ \\
\hline 2nd Parity & $244(27)$ \\
\hline 3rd Parity & $119(13)$ \\
\hline 4th Parity & $24(3)$ \\
\hline 5th Parity & $4(0.4)$ \\
\hline 6th Parity & $1(0.1)$ \\
\hline Parity (not available) & $242(26)$ \\
\hline In vitro fertilization (IVF) & $162(18)$ \\
\hline \multicolumn{2}{|c|}{ Occupation of 918 individuals (positive isolates) } \\
\hline Self-employed & $281(31)$ \\
\hline Army personal & $1(0.1)$ \\
\hline Teacher & $94(10)$ \\
\hline Officer & $197(21)$ \\
\hline Worker & $183(20)$ \\
\hline Farmer & $96(10)$ \\
\hline Student & $3(0.3)$ \\
\hline Healthcare worker & $34(4)$ \\
\hline Engineer & $5(0.5)$ \\
\hline Housewife & $24(3)$ \\
\hline
\end{tabular}

Table 2. Enterobacterales carriage among 3104 samples from pregnant women.

\begin{tabular}{ccccc}
\hline & Total (\%), $\boldsymbol{n = 3 1 0 4}$ & $\begin{array}{c}\text { ESBL-Positive } \\
\boldsymbol{n} \mathbf{( \% )}\end{array}$ & $\begin{array}{c}\text { ESBL-Negative } \\
\boldsymbol{n} \mathbf{( \% )}\end{array}$ & $\boldsymbol{p}$-Value \\
\hline E. coli & $667(21)$ & $340(11)$ & $327(11)$ & NS \\
K. pneumoniae & $243(8)$ & $88(3)$ & $155(6)$ & NS \\
K. oxytoca & $8(0.3)$ & $4(0.1)$ & $4(0.1)$ & NS \\
\hline & $918(30)$ & $432(14)$ & $486(16)$ & NS \\
\hline
\end{tabular}

NS: non-significant; ESBL = extended-spectrum beta-lactamase.

\subsection{ESBL-E and Antibiotic Resistance}

The ESBL-E isolates identified in this study showed antibiotic resistance to different classes of antibiotics, as summarized in Table 3. Of the 918 ESBL-Enterobacterales isolates, mostly from E. coli and Klebsiella spp., $97 \%(n=891 / 918)$ showed antibiotic resistance to at least one antimicrobial agent. Due to nonuniformity in the availability of susceptibility data, only resistance data with complete antimicrobial susceptibility for ampicillin, ceftriaxone, ertapenem, gentamicin, ciprofloxacin and trimethoprim/sulfamethoxazole $(n=904 / 918)$ were considered for better comparability (Table 3). In general, the ESBL-positive isolates showed higher antibiotic resistance compared to the ESBL-negative ones. Only 8\% (72/904) were phenotypically resistant to carbapenem. Resistance to multiple antibiotics was common; the prevalence of multidrug resistance (MDR) was higher in ESBL-positive isolates (65\% vs. $39 \%, p<0.001$, Table 3$)$. 
Table 3. Phenotypic antibiotic resistance of Enterobacterales from vaginal swabs of Vietnamese pregnant women 2016-2020 ( $n=904)$.

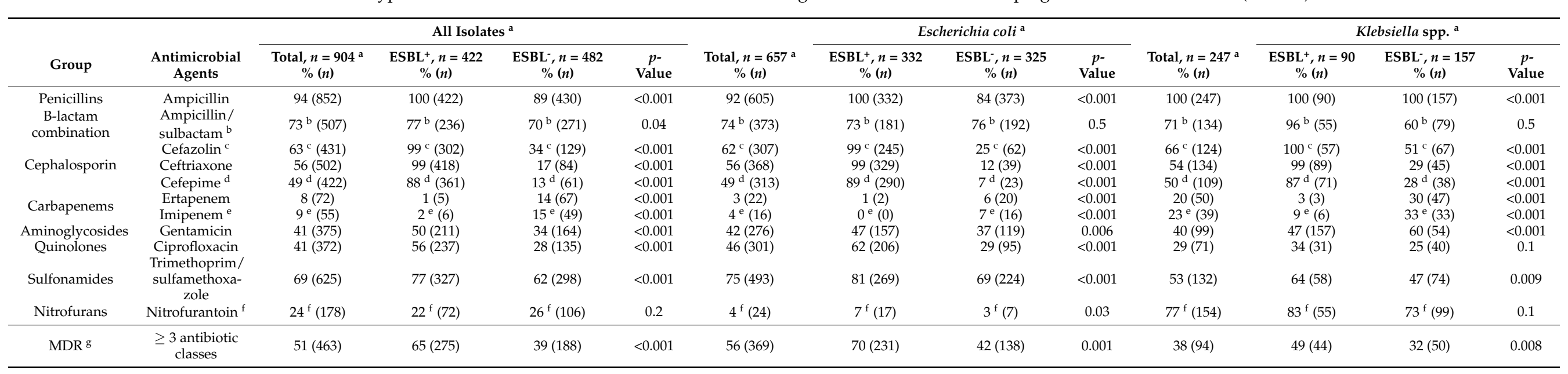

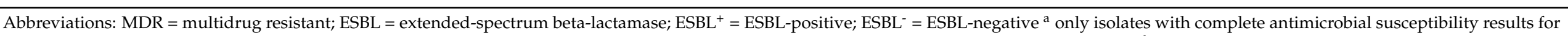

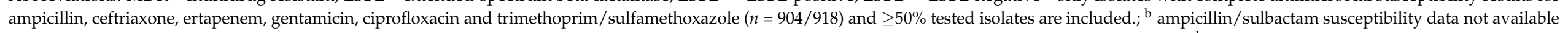

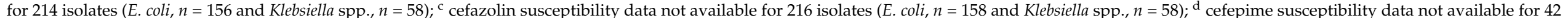

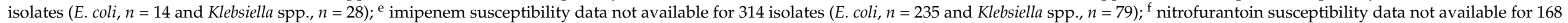
isolates (E. coli, $n=122$ and Klebsiella spp., $n=46$ ), ${ }^{\mathrm{g}}$ multi-drug resistance (MDR) is defined as resistance to three of more classes of antibiotics. All beta-lactams were considered as one class. 
Of the 667 E. coli isolated from the vaginal swabs, 51\% (340/667) were ESBL producers. MDR was frequent in ESBL-positive E. coli than ESBL-negative E. coli ( $70 \%$ vs. $42 \%$, $p=0.001)$. Co-resistances to gentamicin, ciprofloxacin and trimethoprim/sulfamethoxazole were frequent. Only $3 \%$ (22/657) of E. coli tested were resistant to carbapenem (Table 3).

Over $50 \%$ of Klebsiella spp. collected from the vaginal swabs were beta-lactam resistant. Of 243 K. pneumoniae, 36\% (88/243) and of 8 K. oxytoca, 50\% (4/8) were ESBL-producers. Like E. coli, MDR was more frequent in ESBL-producers ( $49 \%$ vs. $32 \%, \mathrm{p}=0.008)$. In ESBLproducers, the prevalence of carbapenem resistance was low (3\% towards ertapenem and $9 \%$ towards imipenem). Surprisingly, a significantly higher percentage of ESBL-negative Klebsiella spp. were carbapenem-resistant (30\% towards ertapenem and 33\% towards imipenem, Table 3).

\section{Discussion}

Maternal vaginal colonization is an important risk factor in pregnant women, and ESBL-producing Enterobacterales can transmit pathogens to the newborns [16]. There is limited information on reproductive tract infections in resource-poor settings, such as Vietnam. To the best of our knowledge, this is the first study, which aimed to investigate the incidence of ESBL-producing Enterobacterales and to understand antibiotic resistance to commonly prescribed drugs.

In the present study, the prevalence of vaginal Enterobacterales and ESBL-Enterobacterales colonization among pregnant women was 30\% and 10\%, respectively. The ESBL-E incidence observed in this study is higher than other upper-middle-income countries, such as Norway (3\%) [15] and Argentina (5\%) [17], but lower than in other African countries $(17 \%)$, respectively [18]. Nonuniform approaches to study design, particularly differences in inclusion criteria, age groups, the inclusion of hospital and/or community populations, etc., may modulate the reported ESBL incidences across geographic regions [19]. In addition, using standardized and harmonized AMR sampling, detection and interpretation procedures may additionally contribute to the differences in ESBL-E incidences.

In this study, E. coli and K. pneumoniae are the most commonly isolated gram-negative Enterobacterales and were known to be the major causative agents of neonatal ESBL-E infections $[20,21]$. Furthermore, these strains are reported to be the predominant uropathogenic bacteria in pregnant women in developing countries in Africa and Asia [22] and are acknowledged as common causes of community and hospital-acquired bacteremia in adults in Vietnam [23,24]. The Global Antimicrobial Surveillance System (GLASS) lists E. coli and $K$. pneumoniae as the two most common resistant priority pathogens $[1,25]$. The vaginal $E$. coli colonization rate of $21 \%$ observed in this study is a risk factor for preterm birth [26]. Vaginal E. coli colonization is associated with obstetric infections and neonatal infections. The colonization rate was higher than other studies, e.g., 20\% in Lithuania [27], 14\% in Argentina [28]. The $8 \%$ rate of K. pneumoniae isolates observed in this study is lower than those reported from Bangladesh (9\%) [29] and in Nigeria (15\%) [30]. The rates of ESBL-E producing E. coli and K. pneumoniae vary depending on the regions [31] and ESBL producing E. coli in this study was $11 \%$, which is significantly higher [28].

In the presented study, the Enterobacterales isolates were observed to be more resistant to commonly prescribed drugs, such as ciprofloxacin ( $41 \%)$, trimethoprim/sulfamethoxazole $(69 \%)$, and third-generation cephalosporins (56\%, suggesting that these drugs may not be effective in treating infections in pregnant women. Ampicillin is the first-line antibiotic for treating obstetric and neonatal infections [32], and resistance to ampicillin is alarming for $E$. coli. A recent review showed a high prevalence of antibiotic resistance to commonly used antibiotics in commensal $E$. coli isolates from humans in low-middle-income countries (LMICs), including Vietnam; ampicillin (72\%), oxytetracycline (78\%), tetracycline $(67 \%)$, streptomycin (58\%) and trimethoprim (67\%), chloramphenicol (45\%), ciprofloxacin (17\%), co-trimoxazole (63), nalidixic acid (30\%) and third-generation cephalosporin-cefotaxime (27\%) [33]. In this study, E. coli resistance to ampicillin was $92 \%$ and $56 \%$ to third-generation cephalosporin, higher than another study [28]. Furthermore, over 50\% of Klebsiella sp. are 
resistant to third-generation cephalosporin, and over 36\% were ESBL-producers, which is in line with previously reported epidemiological data from this region [34]. Unexpectedly, our data indicated that over $20 \%$ of K. pneumoniae and K. oxytoca were carbapenem-resistant, which was much higher than anticipated. The acquisition of carbapenem-resistant Enterobacterales in Vietnam through contact with hospital settings has been described previously [35]. The high rate of carbapenem resistance in Klebsiella spp. is alarming and warrants further investigations.

Most of the commonly prescribed drugs revealed high resistance; drugs such as broad-range penicillin, cephalosporins and carbapenems, may be considered as an alternative treatment option. Carbapenems are considered drugs of choice for infections caused by ESBL-producing enterobacteria [36,37]. However, the increased use of carbapenems for ESBL-K. pneumoniae infections has led to the rapid emergence of carbapenem resistance [38]. Therefore, using carbapenems as empiric antimicrobial therapy should be prudent.

There are a few study limitations. A major limitation of this study is that AMR data were generated in the routine microbiological diagnostic and molecular characterization could not be performed routinely due to limited resources. Since bacterial isolates were not cryopreserved, retrospective genotypic analysis for ESBL-producers could not be performed. Nonetheless, the presented findings highlight the ongoing AMR problem in Vietnam and raise important issues to access molecular typing methods/facilities in low and middle-income countries. Genotypic analysis of the observed drug-resistant isolates would have been valuable to verify the findings. Other limitations include that the study did not examine when pregnant women became colonized with ESBL-E. Second, the study did not examine potential risk factors that promote ESBL-E colonization/infection, which would have allowed for any intervention for clinical management. Finally, the study does not document antibiotics taken as self-medication or prescribed during pregnancy.

\section{Materials and Methods}

\subsection{Ethics}

Informed written consent was obtained from all study participants. The study was approved by the Institutional Review Board of Vietnam Military Medical University, Hanoi, Vietnam. The study was conducted between January 2016 and November 2020 at the National Hospital of Obstetrics and Gynecology (NHOG) in Hanoi, Vietnam, the largest healthcare center and a referral hospital for northern Vietnam, serving a population of $\sim 55$ million people with 450,000 deliveries annually. Vaginal swab samples $(n=3104)$ were collected from hospitalized pregnant women and screened for Gram-negative bacteria. All clinical and demographic parameters are summarized in Table 1.

\subsection{Identification and Antimicrobial Susceptibility Testing}

Vaginal samples were processed for detection of Enterobacterales according to routine laboratory protocols. In brief, vaginal swab samples were inoculated onto MacConkey agar plates (Merck, Kenilworth, NJ, USA) and incubated at $37^{\circ} \mathrm{C}$ for $24 \mathrm{~h}$ to grow Gram-negative enteric bacteria. The identified colonies were subjected to direct identification and antimicrobial susceptibility testing using the VITEK ${ }^{\circledR} 2$ compact automated system (bioMérieux, Lyon, France) with VITEK ${ }^{\circledR} 2$ GN ID card (bioMérieux, Lyon, France) for species-level identification of clinically important Gram-negative bacilli, including a broad range of Gramnegative Enterobacterales, non-Enterobacterales and other highly pathogenic organisms.

\subsection{Identification of ESBL-E Phenotypes}

ESBL phenotypic confirmation was performed using a combined disc diffusion method recommended by Clinical and Laboratory Standards Institute-2018 (CLSI, Wayne, PA, USA) [39]. Briefly, colonies from the vaginal samples were transferred to $1 \mathrm{~mL}$ of normal saline to adjust turbidity to $0.5 \mathrm{McF}$ arland standard. A lawn culture was established with this inoculum on a Mueller-Hinton agar plate. The inoculum was allowed to 
dry for $15 \mathrm{~min}$, and the antibiotic discs cefotaxime $(30 \mathrm{mg}) /$ ceftazidime-clavulanic acid (30 mg/10 mg) and ceftazidime (30 mg)/ceftazidime-clavulanic acid (30 mg/ $10 \mathrm{mg}$ ) were placed $20 \mathrm{~mm}$ apart and incubated for $37^{\circ} \mathrm{C}$ for $24 \mathrm{~h}$. The phenotypic test was interpreted according to CLSI guidelines based on the diameter of the zone of inhibition $(\geq 5 \mathrm{~mm})$. The quality control of antibiotic susceptibility testing was performed with $E$. coli ATCC 25922 and K. pneumoniae ATCC 700603 (for $\beta$-lactam/ $\beta$-lactamase inhibitor combination).

A large proportion of the bacterial isolates were tested for susceptibility to different classes of antibiotics, including $\beta$-lactams (ampicillin, amoxicillin/clavulanic acid, ampicillin/sulbactam, piperacillin/tazobactam), cephalosporins/cephamycins (cefazolin, cefepime, cefotaxime, ceftriaxone, cefuroxime, ceftazidime), carbapenems (doripenem, ertapenem, imipenem, meropenem), aminoglycosides (gentamicin, tobramycin, amikacin), tetracycline, quinolones (ciprofloxacin, levofloxacin, norfloxacin), sulfonamides (trimethoprim/sulfamethoxazole), fosfomycin and nitrofurans (nitrofurantoin) using VITEK ${ }^{\circledR} 2$. In case of unavailability of VITEK ${ }^{\circledR} 2$ test kits, susceptibility testing was performed by disk diffusion.

\subsection{Statistical Analysis}

Statistical analyses were performed using Stata 13.1 (StataCorp, College Station, TX, USA) and R 4.0.2 [40]. All tests were two-tailed, and a $p$-value of 0.05 was considered statistically significant. Comparisons of associations between Enterobacterales carriage, antibiotic susceptibility were computed using a chi-squared test.

\section{Conclusions}

Pregnant women had a high prevalence of colonization and may transmit ESBL-E to neonates at birth, an important risk factor for early-onset neonatal sepsis. However, further studies are needed to validate our findings and to assess the necessity of ESBL screening in pregnant women. The high prevalence of ESBL-producing Enterobacterales and carbapenem resistance in Klebsiella spp. in Vietnam is alarming and highlights the necessity for implementing AMR surveillance in Vietnamese hospitals. Most importantly, access to molecular typing methods in low- and middle-income countries is desperately needed to better understand spreading AMR in the global context.

Author Contributions: N.T.V. and H.A.S. designed the study. T.P.V. contributed to the study design. V.V.D., N.D.T. and N.V.T. collected samples, demographic data, carried out statistical analyses, and interpreted results. H.V.T., N.L.T., C.V.M. were involved in the experimental procedures and data collation. S.R.P. and D.N. analyzed the data and reviewed the manuscript. T.P.V. wrote the manuscript. T.P.V. and D.N. revised and finalized the manuscript. All authors have read and agreed to the published version of the manuscript.

Funding: This research was funded by the Do Xuan Hop Grant of Vietnam Military Medical University.

Institutional Review Board Statement: The study was conducted according to the guidelines of the Declaration of Helsinki, and approved by the Institutional Review Board of Vietnamese Military Medical University, Hanoi, Vietnam (VMMU-IEC-AMR-02-20201603-V2).

Informed Consent Statement: Informed consent was obtained from all subjects involved in the study.

Data Availability Statement: The data supporting reported results are available on request.

Acknowledgments: We thank all study subjects for their participation.

Conflicts of Interest: The authors declare no conflict of interest. The funders had no role in the design of the study; in the collection, analyses, or interpretation of data; in the writing of the manuscript, or in the decision to publish the results. 


\section{References}

1. WHO. Global Priority List of Antibiotic Resistant Bacteria to Guide Research, Discovery, and Development of New Antibiotics; World Health Organization: Geneva, Switzerland, 2017.

2. Trung, N.T.; Hien, T.T.; Huyen, T.T.; Quyen, D.T.; Binh, M.T.; Hoan, P.Q.; Meyer, C.G.; Velavan, T.P.; Song le, H. Simple multiplex PCR assays to detect common pathogens and associated genes encoding for acquired extended spectrum betalactamases (ESBL) or carbapenemases from surgical site specimens in Vietnam. Ann. Clin. Microbiol. Antimicrob. 2015, 14, 23. [CrossRef]

3. Morrissey, I.; Hackel, M.; Badal, R.; Bouchillon, S.; Hawser, S.; Biedenbach, D. A Review of Ten Years of the Study for Monitoring Antimicrobial Resistance Trends (SMART) from 2002 to 2011. Pharmaceuticals 2013, 6, 1335-1346. [CrossRef] [PubMed]

4. Suwantarat, N.; Carroll, K.C. Epidemiology and molecular characterization of multidrug-resistant Gram-negative bacteria in Southeast Asia. Antimicrob. Resist. Infect. Control 2016, 5, 15. [CrossRef] [PubMed]

5. Le, N.K.; Hf, W.; Vu, P.D.; Khu, D.T.K.; Le, H.T.; Hoang, B.T.N.; Vo, V.T.; Lam, Y.M.; Vu, D.T.V.; Nguyen, T.H.; et al. High prevalence of hospital-acquired infections caused by gram-negative carbapenem resistant strains in Vietnamese pediatric ICUs: A multi-centre point prevalence survey. Medicine 2016, 95, e4099. [CrossRef] [PubMed]

6. $\quad$ Peters, L.; Olson, L.; Khu, D.T.K.; Linnros, S.; Le, N.K.; Hanberger, H.; Hoang, N.T.B.; Tran, D.M.; Larsson, M. Multiple antibiotic resistance as a risk factor for mortality and prolonged hospital stay: A cohort study among neonatal intensive care patients with hospital-acquired infections caused by gram-negative bacteria in Vietnam. PLoS ONE 2019, 14, e0215666. [CrossRef]

7. Frank Wolf, M.; Abu Shqara, R.; Naskovica, K.; Zilberfarb, I.A.; Sgayer, I.; Glikman, D.; Rechnitzer, H.; Fleisher Sheffer, V.; Bornstein, J. Vertical Transmission of Extended-Spectrum, Beta-Lactamase-Producing Enterobacteriaceae during Preterm Delivery: A Prospective Study. Microorganisms 2021, 9, 506. [CrossRef] [PubMed]

8. Berg, B.R.; Houseman, J.L.; terSteeg, Z.E.; LeBar, W.D.; Newton, D.W. Antimicrobial susceptibilities of group B streptococcus isolates from prenatal screening samples. J. Clin. Microbiol. 2014, 52, 3499-3500. [CrossRef]

9. Bookstaver, P.B.; Bland, C.M.; Griffin, B.; Stover, K.R.; Eiland, L.S.; McLaughlin, M. A Review of Antibiotic Use in Pregnancy. Pharmacotherapy 2015, 35, 1052-1062. [CrossRef]

10. Jalilian, N.; Kooshkiforooshani, M.; Ahmadi, S.; Nankali, A. Colonisation with extended-spectrum beta-lactamase-producing Enterobacteriaceae in pregnant/post-partum women: Systematic review and meta-analysis. J. Glob. Antimicrob. Resist. 2019, 19, 338-347. [CrossRef] [PubMed]

11. Denisuik, A.J.; Lagace-Wiens, P.R.; Pitout, J.D.; Mulvey, M.R.; Simner, P.J.; Tailor, F.; Karlowsky, J.A.; Hoban, D.J.; Adam, H.J.; Zhanel, G.G.; et al. Molecular epidemiology of extended-spectrum beta-lactamase-, AmpC beta-lactamase- and carbapenemaseproducing Escherichia coli and Klebsiella pneumoniae isolated from Canadian hospitals over a 5 year period: CANWARD 2007-11. J. Antimicrob. Chemother. 2013, 68 (Suppl. S1), i57-i65. [CrossRef]

12. Mendes, R.E.; Mendoza, M.; Banga Singh, K.K.; Castanheira, M.; Bell, J.M.; Turnidge, J.D.; Lin, S.S.; Jones, R.N. Regional resistance surveillance program results for 12 Asia-Pacific nations (2011). Antimicrob. Agents Chemother. 2013, 57, 5721-5726. [CrossRef] [PubMed]

13. Birgy, A.; Mariani-Kurkdjian, P.; Bidet, P.; Doit, C.; Genel, N.; Courroux, C.; Arlet, G.; Bingen, E. Characterization of extendedspectrum-beta-lactamase-producing Escherichia coli strains involved in maternal-fetal colonization: Prevalence of E. coli ST131. J. Clin. Microbiol. 2013, 51, 1727-1732. [CrossRef] [PubMed]

14. Kang, C.I.; Kim, S.H.; Park, W.B.; Lee, K.D.; Kim, H.B.; Kim, E.C.; Oh, M.D.; Choe, K.W. Bloodstream infections due to extendedspectrum beta-lactamase-producing Escherichia coli and Klebsiella pneumoniae: Risk factors for mortality and treatment outcome, with special emphasis on antimicrobial therapy. Antimicrob. Agents Chemother. 2004, 48, 4574-4581. [CrossRef] [PubMed]

15. Rettedal, S.; Lohr, I.H.; Bernhoff, E.; Natas, O.B.; Sundsfjord, A.; Oymar, K. Extended-spectrum beta-lactamase-producing Enterobacteriaceae among pregnant women in Norway: Prevalence and maternal-neonatal transmission. J. Perinatol. 2015, 35, 907-912. [CrossRef]

16. Chan, G.J.; Lee, A.C.; Baqui, A.H.; Tan, J.; Black, R.E. Risk of early-onset neonatal infection with maternal infection or colonization: A global systematic review and meta-analysis. PLoS Med. 2013, 10, e1001502. [CrossRef] [PubMed]

17. Mansouri, F.; Sheibani, H.; Javedani Masroor, M.; Afsharian, M. Extended-spectrum beta-lactamase (ESBL)-producing Enterobacteriaceae and urinary tract infections in pregnant/postpartum women: A systematic review and meta-analysis. Int. J. Clin. Pract. 2019, 73, e13422. [CrossRef] [PubMed]

18. Tansarli, G.S.; Poulikakos, P.; Kapaskelis, A.; Falagas, M.E. Proportion of extended-spectrum beta-lactamase (ESBL)-producing isolates among Enterobacteriaceae in Africa: Evaluation of the evidence-Systematic review. J. Antimicrob. Chemother. 2014, 69, 1177-1184. [CrossRef]

19. Takashima, K.; Wada, K.; Tra, T.T.; Smith, D.R. A review of Vietnam's healthcare reform through the Direction of Healthcare Activities (DOHA). Environ. Health Prev. Med. 2017, 22, 74. [CrossRef]

20. Flokas, M.E.; Karanika, S.; Alevizakos, M.; Mylonakis, E. Prevalence of ESBL-Producing Enterobacteriaceae in Pediatric Bloodstream Infections: A Systematic Review and Meta-Analysis. PLoS ONE 2017, 12, e0171216. [CrossRef]

21. Dramowski, A.; Cotton, M.F.; Rabie, H.; Whitelaw, A. Trends in paediatric bloodstream infections at a South African referral hospital. BMC Pediatrics 2015, 15, 33. [CrossRef]

22. Belete, M.A.; Saravanan, M. A Systematic Review on Drug Resistant Urinary Tract Infection Among Pregnant Women in Developing Countries in Africa and Asia; 2005-2016. Infect. Drug Resist. 2020, 13, 1465-1477. [CrossRef] 
23. Dat, V.Q.; Vu, H.N.; Nguyen The, H.; Nguyen, H.T.; Hoang, L.B.; Vu Tien Viet, D.; Bui, C.L.; Van Nguyen, K.; Nguyen, T.V.; Trinh, D.T.; et al. Bacterial bloodstream infections in a tertiary infectious diseases hospital in Northern Vietnam: Aetiology, drug resistance, and treatment outcome. BMC Infect. Dis. 2017, 17, 493. [CrossRef]

24. Gandra, S.; Alvarez-Uria, G.; Turner, P.; Joshi, J.; Limmathurotsakul, D.; van Doorn, H.R. Antimicrobial Resistance Surveillance in Low- and Middle-Income Countries: Progress and Challenges in Eight South Asian and Southeast Asian Countries. Clin. Microbiol. Rev. 2020, 33. [CrossRef] [PubMed]

25. Tacconelli, E.; Carrara, E.; Savoldi, A.; Harbarth, S.; Mendelson, M.; Monnet, D.L.; Pulcini, C.; Kahlmeter, G.; Kluytmans, J.; Carmeli, Y.; et al. Discovery, research, and development of new antibiotics: The WHO priority list of antibiotic-resistant bacteria and tuberculosis. Lancet Infect. Dis. 2018, 18, 318-327. [CrossRef]

26. Krohn, M.A.; Thwin, S.S.; Rabe, L.K.; Brown, Z.; Hillier, S.L. Vaginal colonization by Escherichia coli as a risk factor for very low birth weight delivery and other perinatal complications. J. Infect. Dis. 1997, 175, 606-610. [CrossRef]

27. Tameliene, R.; Barcaite, E.; Stoniene, D.; Buinauskiene, J.; Markuniene, E.; Kudreviciene, A.; Vitkauskiene, A.; Jomantiene, D.; Nadisauskiene, R. Escherichia coli colonization in neonates: Prevalence, perinatal transmission, antimicrobial susceptibility, and risk factors. Medicina 2012, 48, 71-76. [CrossRef]

28. Villar, H.E.; Aubert, V.; Baserni, M.N.; Jugo, M.B. Maternal carriage of extended-spectrum beta-lactamase-producing Escherichia coli isolates in Argentina. J. Chemother. 2013, 25, 324-327. [CrossRef] [PubMed]

29. Chan, G.J.; Modak, J.K.; Mahmud, A.A.; Baqui, A.H.; Black, R.E.; Saha, S.K. Maternal and neonatal colonization in Bangladesh: Prevalences, etiologies and risk factors. J. Perinatol. 2013, 33, 971-976. [CrossRef] [PubMed]

30. Ekwempu, C.C.; Lawande, R.V.; Egler, L.J. Microbial flora of the lower genital tract of women in labour in Zaria, Nigeria. J. Clin. Pathol. 1981, 34, 82-83. [CrossRef]

31. Jones, R.N.; Flonta, M.; Gurler, N.; Cepparulo, M.; Mendes, R.E.; Castanheira, M. Resistance surveillance program report for selected European nations (2011). Diagn. Microbiol. Infect. Dis. 2014, 78, 429-436. [CrossRef]

32. Saez-Lopez, E.; Guiral, E.; Fernandez-Orth, D.; Villanueva, S.; Gonce, A.; Lopez, M.; Teixido, I.; Pericot, A.; Figueras, F.; Palacio, M.; et al. Vaginal versus Obstetric Infection Escherichia coli Isolates among Pregnant Women: Antimicrobial Resistance and Genetic Virulence Profile. PLoS ONE 2016, 11, e0146531. [CrossRef] [PubMed]

33. Nji, E.; Kazibwe, J.; Hambridge, T.; Joko, C.A.; Larbi, A.A.; Damptey, L.A.O.; Nkansa-Gyamfi, N.A.; Stalsby Lundborg, C.; Lien, T.Q. High prevalence of antibiotic resistance in commensal Escherichia coli from healthy human sources in community settings. Sci. Rep. 2021, 11, 3372. [CrossRef]

34. Wyres, K.L.; Nguyen, T.N.T.; Lam, M.M.C.; Judd, L.M.; van Vinh Chau, N.; Dance, D.A.B.; Ip, M.; Karkey, A.; Ling, C.L.; Miliya T.; et al. Genomic surveillance for hypervirulence and multi-drug resistance in invasive Klebsiella pneumoniae from South and Southeast Asia. Genome Med. 2020, 12, 11. [CrossRef] [PubMed]

35. Tran, D.M.; Larsson, M.; Olson, L.; Hoang, N.T.B.; Le, N.K.; Khu, D.T.K.; Nguyen, H.D.; Vu, T.V.; Trinh, T.H.; Le, T.Q.; et al. High prevalence of colonisation with carbapenem-resistant Enterobacteriaceae among patients admitted to Vietnamese hospitals: Risk factors and burden of disease. J. Infect. 2019, 79, 115-122. [CrossRef] [PubMed]

36. Paterson, D.L.; Bonomo, R.A. Extended-spectrum beta-lactamases: A clinical update. Clin. Microbiol. Rev. 2005, 18, 657-686. [CrossRef]

37. Laupland, K.B.; Church, D.L.; Vidakovich, J.; Mucenski, M.; Pitout, J.D. Community-onset extended-spectrum beta-lactamase (ESBL) producing Escherichia coli: Importance of international travel. J. Infect. 2008, 57, 441-448. [CrossRef] [PubMed]

38. Corbella, X.; Montero, A.; Pujol, M.; Dominguez, M.A.; Ayats, J.; Argerich, M.J.; Garrigosa, F.; Ariza, J.; Gudiol, F. Emergence and rapid spread of carbapenem resistance during a large and sustained hospital outbreak of multiresistant Acinetobacter baumannii. J. Clin. Microbiol. 2000, 38, 4086-4095. [CrossRef] [PubMed]

39. CLSI. CLSI Supplement M100, 28th ed.; Clinical and Laboratory Standards Institute: Wayne, PA, USA, 2018.

40. RStudio, T. RStudio: Integrated Development for R; RStudio, PBC: Boston, MA, USA, 2020. 